\title{
Coronavirus: a shift in focus away from IFN response and towards other inflammatory targets
}

\author{
Akshaya Thoutam $^{1} \cdot$ Mason Breitzig ${ }^{1} \cdot$ Richard Lockey $^{1} \cdot$ Narasaiah Kolliputi $^{1,2}$ (D) \\ Received: 13 July 2020 / Accepted: 19 July 2020 / Published online: 7 September 2020 \\ (C) The International CCN Society 2020
}

\begin{abstract}
In the past two decades, two beta-coronaviruses, severe acute respiratory syndrome-related coronavirus (SARS-CoV-1) and the Middle East respiratory syndrome-related coronavirus (MERS-CoV), have infected approximately 8000 and 2500 across the globe, respectively (de Wit et al. 2016; Amanat and Krammer 2020). The current viral pandemic, caused by SARS-CoV-2, has already affected $4.23 \mathrm{M}$ in less than a year. Of greater concern, the disease caused by SARS-CoV-2, COVID-19, still has a rapidly increasing global burden (Wu et al. 2020; Zhu et al. 2020). To better understand the biology of COVID-19, an initial barrage of studies compared SARS-CoV-2 to other respiratory viruses: MERS-CoV, SARS-CoV-1, human parainfluenza virus 3 (HPIV3), respiratory syncytial virus (RSV), and Influenza A Virus (IAV). These studies indicate that SARS-CoV-2 infected individuals have a consistent chemokine signature comprising cytokines and monocyte-associated chemokines (CCL2 and CCL8). Therefore, it appears that monocyte cytokine production, particularly in those with a diminished innate immunity, is a driving feature of COVID-19 infection.
\end{abstract}

Keywords Interferon regulator factors (IRFs) $\cdot \mathrm{SARS}-\mathrm{CoV}-2 \cdot$ Tocilizumab

Although still fairly new, COVID-19 has quickly caused a global public health crisis and dominated world news. SARSCoV-2 is particularly devastating in those with compromised immune systems, especially older adults whose immune systems are typically diminished due to age and/or the presence of chronic diseases. The weakened innate immunity of elderly individuals leads them to be one of the subpopulations most susceptible to SARS-CoV-2 infection, subsequent development of COVID-19, and a more severe presentation of the disease.

The entry mechanisms and life cycle of SARS-CoV-2 have been explored and indicate that the virus binds to the ACE2 receptor and initiates fusion between the viral membrane and host cell (Astuti and Ysrafil 2020). Pattern Recognition Receptors (PRFs) detect the foreign viral RNA and oligomerize (Janeway Jr. and Medzhitov 2002). This recognition process,

Narasaiah Kolliputi

nkollipu@usf.edu

1 Division of Allergy and Immunology, Department of Internal Medicine, University of South Florida, Tampa, FL 33612, USA

2 Division of Allergy and Immunology, Department of Molecular Medicine, College of Medicine, University of South Florida, Tampa, FL 33612, USA i.e. oligomerization, activates transcriptional factors, such as Interferon Regulator Factors (IRFs) and Nuclear Factor (NF) $\mathrm{kB}$ (Hur 2019), responsible for triggering one of two viral immune responses: 1) induction of Type I and III interferons (IFNI and IFN-III) with the upregulation of Interferon-Stimulated Genes (ISGs), or 2) chemokine secretion that recruits subsets of leukocytes (Lazear et al. 2019; Proudfoot 2002; Sokol and Luster 2015). However, coronaviruses, including SARS-CoV2 , have an array of immuno-evasion characteristics that permit them to effectively avoid these cellular antiviral mechanisms (Astuti and Ysrafil 2020).

Blanco-Melo et al. (2020) compared the transcriptional response of SARS-CoV-2 to that of other viruses. They categorized each viruses' transcriptional responses through a variety of model systems: in vitro tissue culture, ex vivo infection of primary cells, and in vivo samples of COVID-19 patients and animals. Sequencing the polyA RNA of infected cells reveals that A549 lung alveolar cells were non-permissive to SARS$\mathrm{CoV}-2$. These scientists were able to bypass the low expression of the viral receptor ACE2 in A549 cells and map 300x more coverage of the genome $(30 \mathrm{kB})$ via supplementation with ACE2, thus bolstering the rate of SARS-CoV-2 infection. Their western blot and qPCR analyses show elevated nucleocapsid $(\mathrm{N})$ expression and a greater concentration of 
envelope (E) and non-structural protein 14 (nsp14) in cells with a higher presence of ACE2. Additionally, cells treated with universal IFNb show a decrease in viral replication when IFN-I is added, an observation consistent across other studies (Lokugamage et al. 2020). Blanco-Melo et al. (2020) postulate that the high induction of ISG during SARS-CoV-2 infection is independent of IFN-I/-III signals because the induction of cytokines and chemokines is minimally affected by treatment with ruxolitinib. Differential expression analysis show that cells with a high viral replication rate, of SARS-CoV-2 and other viruses, do not produce similar host responses. Even with no significant IFN-I/-III expression, low SARS-CoV-2 multiplicity of infection (MOI) in A549 cells is concomitant with ISG and proinflammatory cytokine signatures. Normal human bronchial epithelial (NHBE) cells typically have a strong chemotactic and inflammatory response with a lack of IFN expression, yet SARS-CoV-2 triggers chemokine signals and pathway responses to IFN-II.

Blanco-Melo et al. (2020) compared postmortem lung samples of male patients, over the age of 60 , with or without COVID-19, to further examine low IFN-I/-III and high chemokine signatures. Genetic analysis reveals an increase in innate and humoral responses. Furthermore, the immune response pathways induced by SARS-CoV-2 infection show high levels of CCL2 and CCL8. They expanded upon their research by obtaining serum samples from two SARS-CoV-2 positive individuals who tested negative for IFNb and IFNl, and found an increase in IL-6, IL-1b, IL1RA, CCL2, CCL8, CXCL2, CXCL8, CXCL9, and CXCL16 levels. Elevation of CXCL9, CXCL16, CCL8, CCL2, and CXCL8 may be the signature of COVID-19 pathology.

In conclusion, the transcriptional footprint of SARS-CoV-2 is different from those of other respiratory viruses. Although a low IFN-I/-III response is observed, evidence suggests there is a consistent chemokine signature. The knowledge derived from the work of Blanco-Melo et al. (2020) may help identify which individuals are at highest risk to develop severe COVID-19. Furthermore, their work demonstrates that induction of CCL2, CCL8, and neutrophils is consistent with the increased neutrophil levels observed in COVID-19 patients. The results of this study also suggest a possible parallel between COVID-19 and cytokine release syndrome. Therefore, tocilizumab should be considered a possible treatment. Additionally, this study offers an explanation as to why subjects with a healthy immune system are typically asymptomatic: hypothesizing that the lack of selective death of Type II pneumocytes prevents fluid leakage and the loss of air function in the lungs. Since research has demonstrated that IFN response is not essential for COVID-19-induced inflammation, studies should review their treatment approach and focus on inflammatory targets other than IFN. Although the study has many strengths, Blanco-Melo et al. (2020) did not focus on specific treatments. Future research should attempt to rapidly develop FDA-approved drugs with immunomodulating properties. The research accomplished by Blanco-Melo et al. (2020) serves as a strong foundation for such studies aimed at inflammatory targets other than IFNs. Data from their work lays the groundwork for the development of immunomodulatory COVID-19 treatments.

Grants N. Kolliputi was funded by the American Heart Association National Scientist Development Grant 09SDG2260957, National Institutes of Health National Heart, Lung, and Blood Institute Grant R01 HL-105932, and the Joy McCann Culverhouse endowment to the Division of Allergy and Immunology.

Author contributions A.T. drafted the manuscript; M.B. edited and revised the manuscript; R.L. and N.K. approved the final version.

\section{Compliance with ethical standards}

Disclosures No conflicts of interest, financial or otherwise, are declared by the authors.

\section{References}

Amanat, F., and Krammer, F. (2020). SARS-CoV-2 Vaccines: Status Report Immunity. Apr 14;52(4):583-589

Astuti I, Ysrafil (2020) Severe acute respiratory syndrome coronavirus 2 (SARS-CoV-2): an overview of viral structure and host response. Diabetes Metab Syndr 14(4):407-412

de Wit E, van Doremalen N, Falzarano D, Munster VJ (2016) SARS and MERS: recent insights into emerging coronaviruses. Nat Rev Microbiol 14:523-534

Hur S (2019) Double-stranded RNA sensors and modulators in innate immunity. Annu Rev Immunol 37:349-375

Janeway CA Jr, Medzhitov R (2002) Innate immune recognition. Annu Rev Immunol 20:197-216

Lazear HM, Schoggins JW, Diamond MS (2019) Shared and distinct functions of type I and type III Interferons. Immunity 50:907-923

Lokugamage, K., Hage, A., Schindewolf, C., Rajsbaum, R., and Menachery, V.D. (2020). SARS-CoV-2 is sensitive to type I interferon pretreatment. bioRxiv.

Blanco-Melo D, Nilsson-Payant B, Liu WC, Uhl S, Hoagland D, Moller R, Jordan T, Oishi K, Panis M, Sachs R, Wang T, et al. (2020). Imbalanced host response to SARS-CoV-2 drives development of COVID-19

Proudfoot AE (2002) Chemokine receptors: multifaceted therapeutic targets. Nat Rev Immunol 2:106-115

Sokol CL, Luster AD (2015) The chemokine system in innate immunity. Cold Spring Harb Perspect Biol 7

Wu F, Zhao S, Yu B, Chen YM, Wang W, Song ZG, Hu Y, Tao ZW, Tian JH, Pei YY, Yuan ML, Zhang YL, Dai FH, Liu Y, Wang QM, Zheng JJ, Xu L, Holmes EC, Zhang YZ (2020) A new coronavirus associated with human respiratory disease in China. Nature 579: 265-269

Zhu N, Zhang D, Wang W, Li X, Yang B, Song J, Zhao X, Huang B, Shi W, Lu R, Niu P, Zhan F, Ma X, Wang D, Xu W, Wu G, Gao GF, Tan W, China Novel Coronavirus Investigating and Research Team (2020) A novel coronavirus from patients with pneumonia in China, 2019. N Engl J Med 382:727-733

Publisher's note Springer Nature remains neutral with regard to jurisdictional claims in published maps and institutional affiliations. 